\title{
Prognostic significance of preoperative serum inflammation markers in patients with male breast cancer
}

\author{
Xing Li ( $D$ lixing@sysucc.org.cn ) \\ Sun Yat-sen University Cancer Center \\ Zhengchong Xiong \\ Sun Yat-sen University Cancer Center \\ Jin Wang \\ Sun Yat-sen University Cancer Center \\ Juan Fu \\ Sun Yat-sen University Cancer Center \\ Xinhua Xie \\ Sun Yat-sen University Cancer Center \\ Xiaoming Xie \\ Sun Yat-sen University Cancer Center
}

\section{Research Article}

Keywords: Male breast cancer, NLR, PLR, LMR, DFS

Posted Date: February 26th, 2021

DOI: https://doi.org/10.21203/rs.3.rs-266906/v1

License: (1) This work is licensed under a Creative Commons Attribution 4.0 International License. Read Full License 


\section{Abstract}

\section{Background}

There were no predictive prognosis factors of serum in male breast cancer, while breast cancer is a heterogeneous disease. The purpose of our study was to determine the prognostic implications of the pretreatment neutrophil-to-lymphocyte ratio (NLR), platelet-to-lymphocyte ratio (PLR) and lymphocyte-tomonocyte ratio (LMR) in the serum of patients with male breast cancer. Methods $\triangle$ We retrospectively identified an random cohort of male breast cancer patients treated at the Sun Yat-Sen University Cancer Center between Jan1, 1996 and Dec31, 2016. A number of 108 patients had a different inflammation markers recorded pre-operation. Survival status was retrieved from our cancer center registry and phone follow-up. Cox proportional hazards regression model were used to analyze the disease-free survival (DFS) and overall survival (OS). Results $\llbracket A m o n g$ these patients in this study, 13 (12\%) had disease recurrence, and 7 (6.5\%) patients appeared distant metastasis. No statistically significant association of the preoperative NLR, PLR or LMR level with patients' different outcomes was found. Conclusions: In short, we were unable to establish a connection between preoperative inflammation biomarkers and male breast cancer patients' survival. Neither NLR, PLR nor LMR is useful for predicting prognosis in male breast cancer patients, and prospective studies to evaluate the above biomarkers as a simple prognostic trail is necessary.

\section{Background}

Human male breast cancer (MBC) is an infrequent cancer and fewer than $1 \%$ of all breast cancers are found in men. ${ }^{[1]}$ Compared with female breast cancer $(F B C)$, researchers have focused relatively little attention on male breast cancer. Therefore, standards treatment for men have usually been derived from clinical trials of female patients. ${ }^{[2]}$ Even in same stage or similar pathological features, the prognosis is usually very unpredictable and heterogeneous. It would be of great value to identify simple and useful markers to stratify male breast cancer patients with high risk and to improve individualized therapy.

Recent study consider that inflammation is a known major driver for the development and progression of cancer. ${ }^{[3]}$ Various immunologic-based score markers, such as neutrophil-to-lymphocyte ratio (NLR) and platelet-to-lymphocyte ratio(PLR), lymphocyte-to-monocyte ratio(LMR) might provide survival information on different cancers, including gastric, colorectal, pancreas, lung, and esophageal cancers. ${ }^{[4-7]}$ Even in female breast cancer, previous meta-analyses have also confirmed that preoperative NLR may be an effective predictive biomarkers for prognosis. ${ }^{[8]}$ The prognostic role of NLR, PLR or LMR in male breast cancer has not been evaluated yet. These blood parameters are easy to perform and inexpensive, and they are readily performed in daily routine. Consequently, the purpose of our study was to evaluate whether the preoperative NLR, PLR or LMR is an independent prognostic indicator in male breast cancer patients.

\section{Methods}


From an institutional database, a number of 108 male patients diagnosed with breast cancer who were undergoing operation at our Cancer Center between 1995 and 2016 were collected in our study. These patients were included in the cohort if they had blood routine examination before their surgery treatment modality. Patients who had the following situation were excluded from this study :1) received preoperative chemotherapy; 2 ) had chronic inflammatory disease including autoimmune disorder and infection ; 3) had distant metastases at diagnosis; 4) had secondary malignancies ; 5) had incomplete clinical pathological data; and 6) lost to follow-up; All patients including in this study received treatment according to the standard treatment guidelines. The data regarding patient-related clinical pathological, such as age, TNM stage, estrogen/progesterone receptor(HR) status, and human epidermal growth factor receptor 2(Her-2) status were collected and analyzed. Our study design was approved by the local institute research ethics committee. Each patient had written informed consent.

The primary endpoint was disease-free survival (DFS), which was defined as the time interval from operation to the date of any recurrence (local, regional, or distant) of breast cancer, or a second primary cancer, or death due to any cause. The secondary end point was overall survival (OS), which was defined as the time interval from diagnosis to death or the last follow-up. We had followed up all patient by medical records review or telephone interview until 1Dec, 2016.

All statistical analyses were performed using SPSS (version 22.0) software. Cox proportional hazards model, including NLR, PLR and LMR were fit to determine these inflammation parameters that were significantly statistically associated with DFS or OS. Binary logistic regression analyses were respectively performed to assess the influence of NLR, PLR and LMR in different groups. Odds Ratio (OR) estimated from logistic regression was reported relative risks with $95 \%$ confidence interval $(95 \% \mathrm{Cl})$. A $p<0.05$ was considered statistically significant.

\section{Results}

\section{Clinicopathological characteristics among the patients of male breast cancer}

We identified 108 male patients who had been diagnosed and underwent breast surgery. the mean age of the patients was $57.7 \pm 13$.9years, with an age distribution of 28-91 years. Tumor size after surgery was classified as pT1-2 in $87.1 \%$, pT3-4 in $8.3 \%$. Lymph node status positive were diagnosed in $33.3 \%$, negative in $66.7 \%$, whereas HR was positive in $75 \%$ of patients, negative in $25 \%$. The Her-2 expression was positive in $20.4 \%$ and negative in $79.6 \%$ patients. Until last follow-up, local recurrence or distant metastasis were confirmed in 20(18.5\%) patients, however there were 42 patients confirmed dead. All patients' characteristics are presented in Table 1. 
Table 1

Clinicopathological characteristics in patients with male breast cancer $(n=108)$

\begin{tabular}{|c|c|c|}
\hline Characteristic & Number & Percent(\%) \\
\hline \multicolumn{3}{|l|}{ Age } \\
\hline Median(range) & $58(28 \sim 91)$ & \\
\hline \multicolumn{3}{|l|}{ Age in years } \\
\hline$<50$ & 31 & 28.7 \\
\hline$\geq 50$ & 77 & 71.3 \\
\hline \multicolumn{3}{|l|}{ T Classification } \\
\hline T1-2 & 94 & 87.1 \\
\hline T3-4 & 9 & 8.3 \\
\hline unknown & 5 & 4.6 \\
\hline \multicolumn{3}{|l|}{ N Classification } \\
\hline NO & 72 & 66.7 \\
\hline N1-3 & 36 & 33.3 \\
\hline \multicolumn{3}{|l|}{ TNM stage } \\
\hline$|+| \mid$ & 83 & 76.9 \\
\hline III & 21 & 19.4 \\
\hline unknown & 4 & 3.7 \\
\hline \multicolumn{3}{|l|}{ HR status } \\
\hline Negative & 27 & 25 \\
\hline Positive & 81 & 75 \\
\hline \multicolumn{3}{|l|}{ HER-2 status } \\
\hline Negative & 86 & 79.6 \\
\hline Positive & 22 & 20.4 \\
\hline
\end{tabular}

2.Comparison of blood parameters among the patients of male breast cancer

The mean preoperative serum NLR, PLR and LMR were $2.15 \pm 0.93$ (range 0.21-4.77) $125.94 \pm$ 58.22(range38.80-440.77) and 4.61 \pm 2.21 (range1.25-13.50), respectively. The median disease-free survival time was 81 months (range 1-287 months). There were13 patients with recurrence, and 7 patients appeared distant metastasis. The average preoperative serum NLR, PLR and LMR levels in patients 
without disease recurrence, with disease recurrence and with metastasis are shown in Fig. 1. There were no significant differences between groups regarding these data.

\section{The prognostic impact of serum NLR, PLR or LMR on survival of male breast cancer}

In the Cox proportional hazards regression model analysis, whether DFS or not OS, the serum NLR or PLR or LMR were not statistically significant with these patients prognosis. Therefore, no multivariate analyses were calculated. They were not independent prognostic indicator in these male patients. This data was respectively summarized in Table 2 and Table 3.

Table 2

Univariate logistic regression model of NLR, PLR or LMR with regard to DFS

\begin{tabular}{|llll|}
\hline Risk factor & $\boldsymbol{\beta}$ & OR $(95 \% \mathrm{Cl})$ & $\boldsymbol{p}$ \\
\hline NLR & -0.291 & $0.747(0.461-1.212)$ & 0.237 \\
\hline PLR & -0.003 & $0.997(0.988-1.005)$ & 0.412 \\
\hline LMR & -0.001 & $0.999(0.876-1.141)$ & 0.992 \\
\hline Abbreviations: & & \\
\hline $\begin{array}{l}\text { Neutrophil/lymphocyte ratio (NLR), platelet/lymphocyte ratio (PLR) lymphocyte/monocyte ratio } \\
\text { (LMR), Cl confidence interval, } \beta \text { regression coefficient. }\end{array}$ & \\
\hline
\end{tabular}

Table 3

Univariate logistic regression model of NLR, PLR or LMR with regard to OS

\begin{tabular}{|llll|}
\hline Risk factor & $\boldsymbol{\beta}$ & OR $(95 \% \mathrm{Cl})$ & $\boldsymbol{p}$ \\
\hline NLR & -0.046 & $0.955(0.774-1.177)$ & 0.664 \\
\hline PLR & 0.000 & $1.000(0.995-1.005)$ & 0.875 \\
\hline LMR & -0.084 & $0.920(0.815-1.038)$ & 0.173 \\
\hline $\begin{array}{l}\text { Abbreviations: Neutrophil/lymphocyte ratio (NLR), platelet/lymphocyte ratio (PLR) } \\
\text { lymphocyte/monocyte ratio (LMR), Cl confidence interval, } \beta \text { regression coefficient. }\end{array}$ & \\
\hline
\end{tabular}

\section{Relationship between different HR or HER-2 status and clinical blood parameters}

Additional analysis was made according to different HR or HER-2 status. Even in subtypes, NLR, PLR or LMR were not a predictive parameter for prognosis. $P$ value for ER or PR positive tumors $(n=81)$, were $0.208,0.517,0.832$ respectively; and for HER2-positive tumors $(n=22)$, $p$ value was $0.180,0.747,0.322$ respectively. These results were summarized in Table 4 and Table 5. In summary, we were unable to establish a connection between preoperative NLR, PLR or LMR and various clinical features, including recurrence, metastasis, HR and HER-2 status. 
Table 4

Univariate logistic regression model of blood parameters ratio with regard to HR status

\begin{tabular}{|c|c|c|c|c|c|c|}
\hline \multirow[t]{3}{*}{ Risk factor } & \multirow{2}{*}{\multicolumn{3}{|c|}{$\begin{array}{l}\text { HR negative } \\
n=27\end{array}$}} & \multirow{2}{*}{\multicolumn{3}{|c|}{$\begin{array}{l}\text { HR positive } \\
n=81\end{array}$}} \\
\hline & & & & & & \\
\hline & $\beta$ & OR $(95 \% \mathrm{Cl})$ & $p$ & $\beta$ & OR (95 \%Cl) & $p$ \\
\hline NLR & -0.184 & $0.832(0.414-1.672)$ & 0.605 & -0.417 & $0.659(0.344-1.261)$ & 0.208 \\
\hline PLR & -0.014 & $0.986(0.954-1.018)$ & 0.389 & -0.003 & $0.997(0.986-1.007)$ & 0.517 \\
\hline LMR & 0.087 & $1.091(0.650-1.833)$ & 0.742 & -0.017 & $0.983(0.843-1.148)$ & 0.832 \\
\hline
\end{tabular}

Table 5

Univariate logistic regression model of blood parameters ratio with regard to HER-2 status

\begin{tabular}{|c|c|c|c|c|c|c|}
\hline & \multirow{2}{*}{\multicolumn{3}{|c|}{$\begin{array}{l}\text { HER-2 negative } \\
\mathrm{n}=86\end{array}$}} & \multirow{2}{*}{\multicolumn{3}{|c|}{$\begin{array}{l}\text { HER-2 positive } \\
n=22\end{array}$}} \\
\hline & & & & & & \\
\hline & $\beta$ & OR $(95 \% \mathrm{Cl})$ & $\mathbf{p}$ & $\beta$ & OR (95 \% Cl) & $\mathbf{p}$ \\
\hline NLR & -0.183 & $0.833(0.52-1.324)$ & 0.440 & -0.887 & $0.412(0.113-1.504)$ & 0.180 \\
\hline PLR & -0.004 & $0.996(0.987-1.006)$ & 0.442 & -0.003 & $0.997(0.982-1.013)$ & 0.747 \\
\hline LMR & -0.035 & $0.966(0815-1.146)$ & 0.691 & 0.168 & $1.183(0.848-1.651)$ & 0.322 \\
\hline
\end{tabular}

\section{Discussion}

Our study was to determine whether inflammatory marker were predictive factors in male breast cancer patients. However, we could not find predictive or prognostic value of NLR, PLR and LMR in this retrospective analysis. Recently, many articles have investigated blood indicators in patients with malignant tumors, the relationship between them is usually a multifactorial and complex process, still poorly understood. They concluded that tumor-associated neutrophil promote remolding of the extracellular matrix, which results in the release of fibroblast growth factor, migration of endothelial cells and the split of tumor cells; in addition, neutrophil-derived reactive oxygen species could inhibit the cytotoxic activity of lymphocytes, reduce the promoting of the extracellular matrix, suppress apoptosis of cancer cells. These events finally enhanced angiogenesis, and tumor growth and influenced survival outcomes in patients with cancer. ${ }^{[9-10]}$ hile lymphocytes play an important role in the immune reaction against tumors, patients with cancer had higher densities of tumor-infiltrating lymphocytes, they could advance responses to treatments and improve outcome. ${ }^{[11-13]}$ As systematic inflammatory markers, 
serum low lymphocyte and high neutrophil, platelet, macrophage counts have been recognized as worse prognosis in solid tumors. When coupled with these indicators, such as NLR, PLR, and LMR, the predictive effect on cancer prognosis may be enhanced. Several studies consistently had found that NLR was an unfavorable prognostic indicator in patients with gynecological, lung, gastrointestinal, and renal cancers. [14-19] A meta-analysis including 8,586 esophageal squamous cell carcinoma patients had reported that high NLR, PLR and low LMR were associated with poorer prognosis. ${ }^{[7]}$

There were also lots of researches in breast cancer, Azab et al studied 465 female breast cancer patients and demonstrated significantly worse prognosis in patients with higher NLR. ${ }^{[20]}$ Several other studies have also shown similar findings. ${ }^{[21,22]} \mathrm{A}$ recent meta-analysis which included eight researches published has shown that higher NLR may associated with poor surviaval. ${ }^{[22-24]}$ It is worth noting that the available data mainly concern female patients.

For the first time, it enrolled an amount of male patients to investigate the prognostic role of these inflammatory markers in male breast cancer patients. In our study, we identified 108 male patients who were diagnosed and underwent breast surgery, after mean follow-up of 86 months, we found that whether DFS or not OS, the serum NLR or PLR or LMR were not statistically significant with these patients prognosis. This was not the same as in the women's study. As breast cancer is a complicated and heterogeneous disease, lots of clinical parameters or biomarkers have been confirmed to be associated with the prognosis of patients, such as hormone status, Her-2 status, and TNM stage. Studies had found that NLR, PLR, and LMR were just systemic inflammatory response related markers and may affect the prognosis in different cancers. In some case, these inflammatory markers may even contradict each other. Subsequently, we did a subgroup analysis, whether in HR positive group nor in HR negative group, we could not find these marks related to the prognosis of patients. It was different in female patients, Orditura $\mathrm{M}$ et al showed that higher NLR could lead to worse prognosis in female patients with early breast cancer, ${ }^{[8]}$ while Yuka Asano et al reported that lower NLR may cause higher efficacy and better

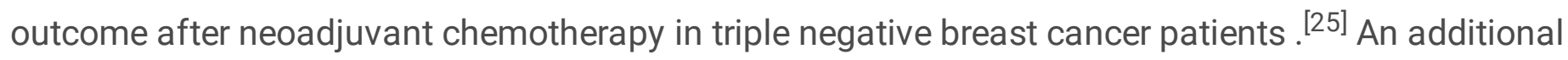
analysis was made according to different HER-2 status, even in different groups we could not identify NLR, PLR, LMR as a predictive factor for prognosis. For male breast cancer patients is rare, treatment standards or prognostic indicators for them have generally been derived from female patients. However, breast cancer is a highly heterogeneous disease, some inflammatory biomarkers could predict the prognosis of patients with a woman, not suitable for male patients. Moreover, gender differences may affect patient preferences and survival factors. Therefore, it could need more studies independent in male patients to improve their therapy and prolong survival.

There are some limitations in our analysis. Firstly, this is a retrospective study with manual data extraction and analysis. However, data concerning laboratory values and survival data were not missed. Secondly, this was a mono-center study, all male patients eligible were included, it may also have the risk of a patient selection bias. Furthermore, serum samples of patients were collected uniformly before treatment to avoid false blood parameters. However, in our study, the number of patients and events were 
relatively small and did not allow to comprehensive multivariable analyze and preclude definitive conclusions, further multiple center and prospective studies still required.

\section{Conclusions}

Although the systemic inflammatory response is closely related to cancer, especially serum NLR or PLR may have clinical role in predicting survival in various cancers. However, this retrospective study failed to show an impact of NLR, PLR, LMR on prognosis in male breast cancer patients. Due to the different influencing factors of hematological components measurement and the heterogeneity of breast cancer, the role of these inflammation markers in male breast cancer should be further evaluated.

\section{Declarations}

\section{Ethics approval and consent to participate}

All procedures performed in this study involving human participants were approved by Ethics Committees of Sun Yat-Sen University Cancer Center and were in accordance with the 1964 Helsinki declaration and its later amendments or comparable ethical standards.

\section{Consent for publication}

Each participant patient had written informed consent about the researchable use of the clinical data.

\section{Availability of data and materials}

The datasets used and/or analysed during the current study are available from the corresponding author on reasonable request.

\section{Competing interests}

The authors declare no conflict of interest.

\section{Funding}

The study was supported by funds from the National Natural Science Foundation of China (81302318 to Xinhua Xie; 81272514,81472575 and 81372133 to Xiaoming Xie).

\section{Authors' contributions}

$X L$ and JW conceived and designed the study, drafted the paper. HLT, JF, and YLK documented and analyzed the data. XMX designed the study and reviewed the paper. XHX interpreted the results and wrote the paper.

\section{Acknowledgments}


We thank the patients who participate in this study. This work was supported by funds from the National Natural Science Foundation of China and the Science and Technology Planning Projects of Guangdong.

\section{References}

1. Fentiman IS, Fourquet A, Hortobagyi GN: Male breast cancer. Lancet 2006; 367:595-604.

2. Harlan LC, Zujewski JA, Goodman MT, Stevens JL: Breast cancer in men in the United States: a population-based study of diagnosis, treatment, and survival. Cancer 2010; 116: 3558-3568.

3. Munn LL: Cancer and inflammation. Wiley Interdiscip Rev Syst Biol Med 2017; 9(2):e1370.

4. Templeton AJ, Pezaro C, Omlin A, McNamara MG, Leibowitz-Amit R, Vera-Badillo FE, et al: Simple prognostic score for metastatic castration-resistant prostate cancer with incorporation of neutrophilto-lymphocyte ratio. Cancer 2014; 120:3346-52.

5. Stotz M, Gerger A, Eisner F, Szkandera J, Loibner H, Ress AL, et al: Increased neutrophil-lymphocyte ratio is a poor prognostic factor in patients with primary operable and inoperable pancreatic cancer. Br J Cancer 2013; 109: 416-21, 2013.

6. Diem S, Schmid S, Krapf M ,Flatz L, Born D, Jochum W, et al: Neutrophil-to-Lymphocyte ratio (NLR) and Platelet-to-Lymphocyte ratio (PLR) as prognostic markers in patients with non-small cell lung cancer (NSCLC) treated with nivolumab. Lung Cancer 2017; 111: 176-181.

7. Yugui Sun, Lifei Zhang: The clinical use of pretreatment NLR, PLR, and LMR in patients with esophageal squamous cell carcinoma: evidence from a meta-analysis. Cancer Management and Research 2018; 10: 6167-6179.

8. Orditura M, Galizia G, Diana A, Saccone C, Cobellis L, Ventriglia J, et al: Neutrophil to lymphocyte ratio (NLR)for prediction of distant metastasis-free survival (DMFS) in early breast cancer:a propensity score-matched analysis. ESMO Open 2016;1(2):e000038.

9. Mantovani A, Allavena P, Sica A, Balkwill F: Cancer-related inflammation. Nature 2008;454:436-44.

10. Rodriguez PC, Ernstoff MS, Hernandez C, Atkins M, Zabaleta J, Sierra R, et al: Arginasel-producing myeloid-derived suppressor cells in renal cell carcinomaare a subpopulation of activated granulocytes. Cancer Res 2009; 69:1553-60.

11. Loi S, Sirtaine N, Piette F, Salgado R, Viale G, Van Eenoo F, et al: Prognostic and predictive value of tumor infiltrating lymphocytes in a phase III randomized adjuvant breast cancer trial in node-positive breast cancer comparing the addition of docetaxel to doxorubicin with doxorubicin-based chemotherapy: BIG 02-98. J Clin Oncol 2013;31:860-7.

12. Gooden MJ, de Bock GH, Leffers N, Daemen T, Nijman HW: The prognostic influence of tumorinfiltrating lymphocytes in cancer: a systematic review with meta-analysis. $\mathrm{Br} \mathrm{J}$ Cancer 2011; 105:93103.

13. West NR, Milne K, Truong PT, Macpherson N, Nelson BH, Watson PH: Tumor-infiltrating lymphocytes predict response to anthracycline-based chemotherapy in estrogen receptor-negative breast cancer. Breast Cancer Res 2011;13: R126. 
14. Templeton AJ, McNamara MG, Šeruga B, Vera-Badillo FE, Aneja P, Ocaña A, et al. Prognostic role of neutrophil-to-lymphocyte ratio in solid tumors: a systematic review and meta-analysis. J Natl Cancer Inst 2014; 106:124.

15. Shimada H, Takiguchi N, Kainuma O, Soda H, Ikeda A, Cho A, et al. High preoperative neutrophillymphocyte ratio predicts poor survival in patients with gastric cancer. Gastric Cancer 2010; 13:1706.

16. Tomita M, Shimizu T, Ayabe T, Yonei A, Onitsuka T. Preoperative neutrophil to lymphocyte ratio as a prognostic predictor after curative resection for non-small cell lung cancer. Anticancer Res 2011; 31:2995-8.

17. Lee YY, Choi CH, Kim HJ, Kim TJ, Lee JW, Lee JH, et al. Pretreatment neutrophil:lymphocyte ratio as a prognostic factor in cervical carcinoma. Anticancer Res 2012; 32:1555-61.

18. Sarraf KM, Belcher E, Raevsky E, Nicholson AG, Goldstraw P, Lim E. Neutrophil/lymphocyte ratio and its association with survival after complete resection in non-small cell lung cancer. $J$ Thorac Cardiovasc Surg 2009; 137:425-8.

19. Ohno Y, Nakashima J, Ohori M, Gondo T, Hatano T, Tachibana M. Follow up of neutrophiltolymphocyte ratio and recurrence of clear cell renal cell carcinoma. J Urol 2012; 187:411-17.

20. Noh $\mathrm{H}$, Eomm M, Han A. Usefulness of pretreatment neutrophil to lymphocyte ratio in predicting disease-specific survival in breast cancer patients. J Breast Cancer 2013; 16(1):55-9.

21. Azab B, Bhatt VR, Phookan J, Murukutla S, Kohn N, Terjanian T, et al. Usefulness of the neutrophil-tolymphocyte ratio in predicting short- and long-term mortality in breast cancer patients. Ann Surg Oncol 2012; 19(1):217-24.

22. Dirican A, Kucukzeybek BB, Alacacioglu A, Kucukzeybek Y, Erten C, Varol U, et al. Do the derived neutrophil to lymphocyte ratio and the neutrophil to lymphocyte ratio predict prognosis in breast cancer? Int J Clin Oncol 2015;20(1):70-81.

23. Chen J, Deng Q, Pan Y, He B, Ying H, Sun $H$, et al. Prognostic value of neutrophil-to-lymphocyte ratio in breast cancer. FEBS Open Bio 2015; 5:502-7.

24. Cihan YB, Arslan A, Cetindag MF, Mutlu H. Lack of prognostic value of blood parameters in patients receiving adjuvant radiotherapy for breast cancer. Asian Pac J Cancer Prev 2014; 15:4225-31.

25. Yuka Asano, Shinichiro Kashiwagi, Naoyoshi Onoda, Satoru Noda, Hidemi Kawajiri, Tsutomu Takashima, et al. Predictive value of neutrophil/lymphocyte ratio for efficacy of preoperative chemotherapy in triple-negative breast cancer. Ann Surg Oncol 2016; 23:1104-1110.

\section{Figures}




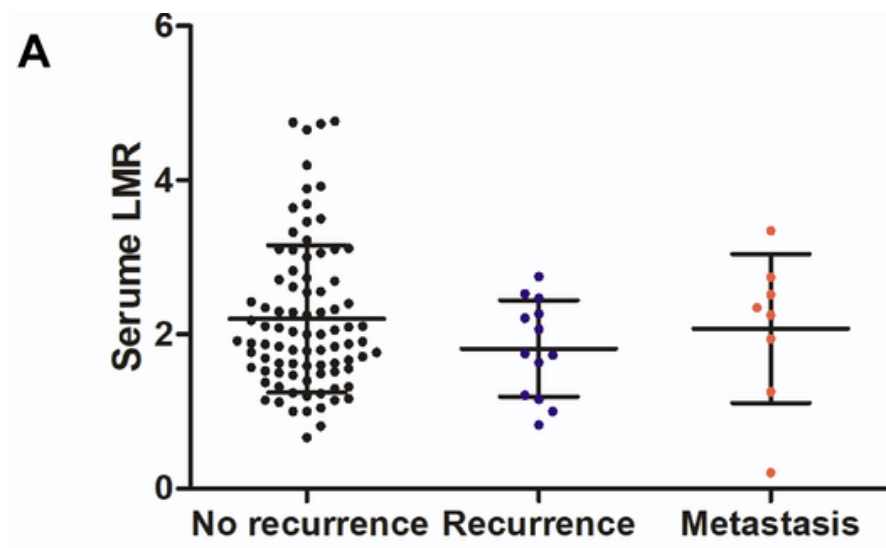

Average $2.20 \pm 0.96 \quad 1.82 \pm 0.63 \quad 2.07 \pm 0.97$

B

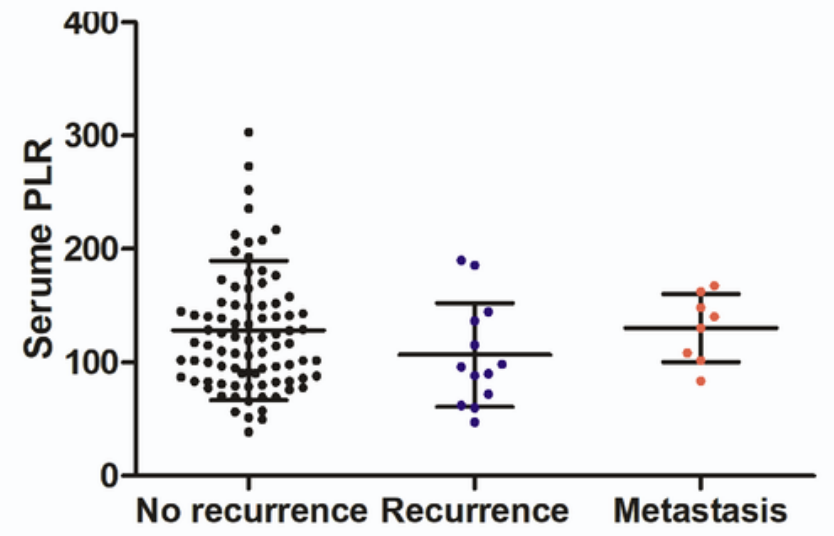

Average $128.40 \pm 61.53 \quad 106.73 \pm 45.86 \quad 130.49 \pm 30.04$

C

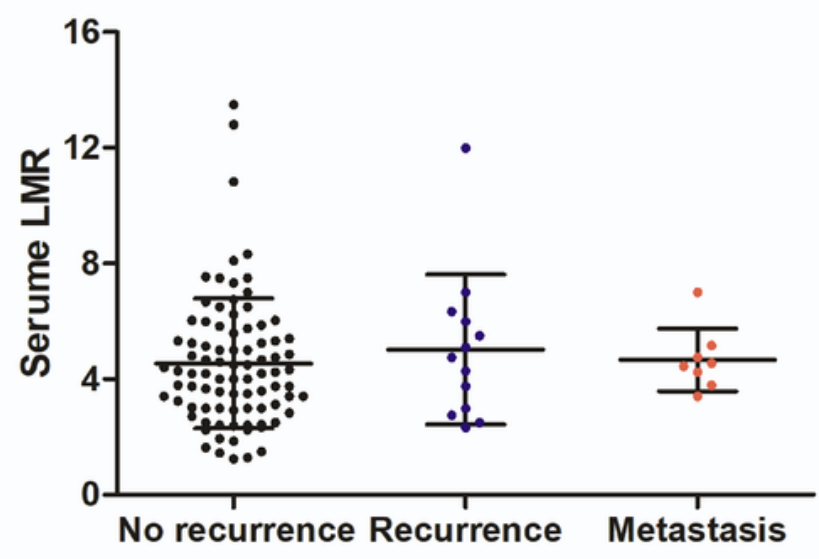

Average $4.55 \pm 2.25 \quad 5.02 \pm 2.59 \quad 4.67 \pm 1.09$

\section{Figure 1}

The average preoperative level of serum NLR (A), PLR (B) and LMR(C) in patients without disease recurrence, with disease recurrence and in patients with metastasis 